PROCEEDINGS OF THE

AMERICAN MATHEMATICAL SOCIETY

Volume 134, Number 1, Pages 177-186

S 0002-9939(05)08325-5

Article electronically published on August 11, 2005

\title{
UNIVERSAL APPROXIMATION OF SYMMETRIZATIONS BY POLARIZATIONS
}

\author{
JEAN VAN SCHAFTINGEN
}

(Communicated by David S. Tartakoff)

\begin{abstract}
Any symmetrization (Schwarz, Steiner, cap or increasing rearrangement) can be approximated by a universal sequence of polarizations which converges in $\mathrm{L}^{p}$ norm for any admissible function in $\mathrm{L}^{p}$ for $1 \leq p<+\infty$ and uniformly for admissible continuous functions. A new Pólya-Szegö inequality is proved for the increasing rearrangement.
\end{abstract}

\section{INTRODUCTION}

A symmetrization $*$ (or rearrangement) maps any function $u: \Omega \rightarrow \mathbb{R}$ to a more symmetrical function $u^{*}: \Omega^{*} \rightarrow \mathbb{R}$. Under some technical assumptions, it has the following properties:

$$
\begin{gathered}
\int_{\Omega} f(u) d x=\int_{\Omega^{*}} f\left(u^{*}\right) d x, \\
\int_{\Omega^{*}}\left|u^{*}-v^{*}\right|^{p} d x \leq \int_{\Omega}|u-v|^{p} d x, \\
\int_{\Omega}|\nabla u|^{p} d x \leq \int_{\Omega^{*}}\left|\nabla u^{*}\right|^{p} d x .
\end{gathered}
$$

Rearrangements are used to prove the symmetry and the existence of solutions of some variational problems.

The symmetrization is defined for sets before being extended to functions. The inequalities (C) and ( $\mathrm{HL}$ are straightforward consequences of the preservation of the inclusions and of the measure of sets by the rearrangement of sets. Pólya-Szegö's inequality ( $\mathrm{PS}$ ) involves the gradient, and a proof that directly uses the definition form rearrangement of sets relies on an isoperimetric inequality for sets and on the coarea formula. The inequality $(\overline{\mathrm{PS}})$ can also be proved by approximation by polarizations, as Brock and Solynin did [5] and as we do in Corollary 6.3. Lieb and Loss [8] and Baernstein [3] deduced it from Riesz-like inequalities that they obtained by approximation.

The first approximation of symmetrizations by simpler symmetrizations appeared in the proof of the classical isoperimetric theorem. A well-chosen sequence of Steiner symmetrization of a convex body converges with respect to the Hausdorff distance to a ball of the same volume. Mani-Levitska proved that random

Received by the editors May 13, 2003 and, in revised form, July 15, 2004.

2000 Mathematics Subject Classification. Primary 26D10; Secondary 28D05, 46E30.

The author is a research fellow of the Fonds National de la Recherche Scientifique (Belgium).

(C)2005 American Mathematical Society Reverts to public domain 28 years from publication 
sequences of Steiner symmetrizations converge [9. Brascamp, Lieb and Luttinger approximated in measure the Schwarz symmetrization of sets by lower order symmetrizations in order to prove a generalized Riesz rearrangement inequality [4, 8]. Sarvas approximated the symmetrization of sets by spherical cap and Steiner symmetrizations [11, while Baernstein [3, Brock and Solynin used polarizations [5]. This result was extended to the cap symmetrization by Smets and Willem [12].

For all the methods of approximations of symmetrizations by polarizations cited above, the sequence of polarizations depends of the function that has to be symmetrized. Our main result (Theorem 4.4) is that there exists a sequence that does not depend on the function or on the function space, and that the increasing rearrangement can also be approximated by polarizations. This symmetrization coincides in the one-dimensional case, with the rearrangement introduced by Carbou [6] and studied by Alberti [1]. The increasing rearrangement inequalities allow us to prove the existence of solutions of variational problems that increase in some direction. Badiale obtained with the moving plane method similar results concerning the monotonicity of solutions to some elliptic systems [2].

By the same method, we prove that cap and Steiner symmetrizations approximate higher-order Steiner and cap symmetrization. The approximating symmetrizations can be of any order, but they must be compatible with the symmetrization that they approximate.

The definitions and basic properties of symmetrizations (section 21) and of polarizations (section 3) are recalled. Section 4 is devoted to the proof of the main result. Finally the method is briefly extended to approximation by symmetrization (section 5), and a Pólya-Szegö inequality is proven (section 6).

\section{Symmetrizations}

Lebesgue's outer on $\mathbb{R}^{N}$ is denoted by $\mathcal{L}^{N}$, Hausdorff's $k$-dimensional outer measure on $\mathbb{R}^{N}$ by $\mathcal{H}^{k}$, the scalar product between $x$ and $y$ by $x \cdot y$, and the Euclidean norm by $|x|=\sqrt{x \cdot x}$. If $x \in \mathbb{R}^{N}, 0 \leq r \leq+\infty$, then

$$
B(p, r)=\left\{x \in \mathbb{R}^{N}|| x-p \mid<r\right\} .
$$

The characteristic function of a set $A$ is denoted by $\chi_{A}$, and the symmetric difference of the sets $A$ and $B$ is denoted by $A \Delta B=(A \backslash B) \cup(B \backslash A)$.

Definition 2.1. If $T$ is a proper affine subspace of $\mathbb{R}^{N}$, the Steiner symmetrization of a set $A \subset \mathbb{R}^{N}$ with respect to $T$ is the unique set $A^{T}$ for which the following holds: for any $p \in T$, if $L$ is the maximal affine subspace orthogonal to $T$ that contains $p$, then

$$
A^{T} \cap L=B(p, r) \cap L,
$$

where $r$ is defined such that $\mathcal{H}^{N-\operatorname{dim} T}(B(p, r) \cap L)=\mathcal{H}^{N-\operatorname{dim} T}(A \cap L)$.

Remark 2.2. The Schwarz symmetrization with respect to $p \in \mathbb{R}^{N}$ is the Steiner symmetrization with respect to $\{p\}$; it is also sometimes the Steiner symmetrization with respect to a straight line [8, 10].

Definition 2.3. A set $S \subset \mathbb{R}^{N}$ is a closed half affine subspace of $\mathbb{R}^{N}$ if it is a closed affine halfspace with respect to its affine span. The boundary of $S$ with respect to its affine span is denoted $\partial S$ and its dimension is $\operatorname{dim} S=\operatorname{dim} \partial S+1$. 
Definition 2.4. If $S$ is a closed half affine subspace $\mathbb{R}^{N}$ and $0<\operatorname{dim} S<N$, the cap symmetrization of a set $A$ with respect to $S$ is the unique set $A^{S}$ for which the following holds: $A^{S} \cap \partial S=A \cap \partial S$ and, for any $q \in \partial S$ and any $s>0$, if $L$ is the maximal affine subspace orthogonal to $\partial S$ that contains $q$, and $p$ is the only point in the intersection $S \cap(L \cap \partial B(q, s))$, then

$$
A^{S} \cap(L \cap \partial B(q, s))=B(p, r) \cap(L \cap \partial B(q, s)),
$$

where $0 \leq r \leq+\infty$ is defined by

$$
\mathcal{H}^{N-\operatorname{dim} S}(B(p, r) \cap(L \cap \partial B(q, s)))=\mathcal{H}^{N-\operatorname{dim} S}(A \cap(L \cap \partial B(q, s))) .
$$

Definition 2.5. Let $A \subseteq \mathbb{R}^{N}$ and $v \in \mathbb{R}^{N} \cap \partial B(0,1)$,

$$
c_{v}(A)=\mathcal{H}^{1}(\{x \in A \mid v \cdot x>0\})-\mathcal{H}^{1}\left(\left\{x \in \mathbb{R}^{N} \backslash A \mid v \cdot x \leq 0\right\}\right)
$$

if the formula has sense and $c_{v}(A)=-\infty$ otherwise.

Definition 2.6. The increasing rearrangement of $A \subset \mathbb{R}^{N}$ with respect to $v \in$ $\mathbb{R}^{N} \cap \partial B(0,1)$ is the unique set $A^{(v, \infty)}$ such that for any $x \in \mathbb{R}^{N}$,

$$
(x+v \mathbb{R}) \cap A^{(v, \infty)}=\left\{y \in(x+v \mathbb{R}) \mid v \cdot y>c_{v}(A \cap(x+v \mathbb{R}))\right\} .
$$

In the sequel, $*$ indifferently denotes a Steiner or cap symmetrization, or an increasing rearrangement.

For any sets $A, B \subseteq \mathbb{R}^{N}$,

$$
A \subseteq B \Longrightarrow A^{*} \subseteq B^{*} .
$$

Proposition 2.7. If $A \subset \mathbb{R}^{N}$ is measurable, then $A^{*}$ is measurable.

Proof. If $*$ is the increasing rearrangement with respect to $v \in \mathbb{R}^{N} \cap \partial B(0,1), A^{*}$ can be written by definition as

$$
A^{*}=\left\{x \in \mathbb{R}^{N} \mid v \cdot x>c(A \cap(x+v \mathbb{R}))\right\} .
$$

Fubini's theorem implies that the function $x \mapsto c_{v}(A \cap(x+v \mathbb{R}))$ is measurable. Hence $A^{*}$ is measurable. The proof is similar for the Steiner and cap symmetrizations.

Definition 2.8. A set $A$ is admissible for a Steiner or cap symmetrization $*$ if $A$ is measurable, and $\mathcal{L}^{N}(A)<+\infty$. If $*$ is the increasing rearrangement with respect to $v, A$ is admissible if and only if

$$
\mathcal{L}^{N}\left(A \Delta\left\{x \in \mathbb{R}^{N} \mid v \cdot x>0\right\}\right)<\infty .
$$

If $A \subset B \subset \mathbb{R}^{N}$ are admissible sets, then

$$
\mathcal{L}^{N}\left(B^{*} \backslash A^{*}\right)=\mathcal{L}^{N}(B \backslash A) .
$$

When the sets $A$ and $B$ may have infinite measure, which is the case for the increasing rearrangement, the second condition is more restrictive than the preservation of the measure of sets $\left(\mathcal{L}^{N}(A)=\mathcal{L}^{N}\left(A^{*}\right)\right)$. If $A, B \subset \mathbb{R}^{N}$ are admissible sets, then

$$
\mathcal{L}^{N}\left(B^{*} \backslash A^{*}\right) \leq \mathcal{L}^{N}(B \backslash A) .
$$

Notation 2.9. For any function $u: \Omega \rightarrow \mathbb{R}$ and $c \in \mathbb{R}$, we write

$$
\{u>c\}=\{x \in \Omega \mid u(x)>c\} .
$$

Definition 2.10. The symmetrization of a function $u: \mathbb{R}^{N} \rightarrow \mathbb{R}$ is, for $y \in \mathbb{R}^{N}$,

$$
u^{*}(y)=\sup \left\{c \in \mathbb{R} \mid y \in\{u>c\}^{*}\right\} .
$$


Proposition 2.11. If $*$ is a rearrangement and $u: \mathbb{R}^{N} \rightarrow \mathbb{R}$ is measurable, then $u^{*}$ is measurable.

Proof. Since $*$ is monotone on sets, $\left\{u^{*}>c\right\}=\bigcup_{n \geq 1}\{u>c+1 / n\}^{*}$, and the conclusion comes from Proposition 2.7

Definition 2.12. A function $u: \Omega \rightarrow \mathbb{R}$ is admissible if for any essinf $u<c<$ ess $\sup u,\{u>c\}$ is admissible.

Definition 2.13. If $*$ is a Steiner or cap symmetrization, if $1 \leq p<+\infty$, let $\mathrm{L}_{*}^{p}\left(\mathbb{R}^{N}\right)=\mathrm{L}_{+}^{p}\left(\mathbb{R}^{N}\right)$ be the set of nonnegative functions of $\mathrm{L}^{p}\left(\mathbb{R}^{N}\right)$, let $\mathrm{C}_{*}\left(\mathbb{R}^{N}\right)=$ $\mathrm{C}_{0,+}\left(\mathbb{R}^{N}\right)$ be the set of nonnegative continuous functions whose limit at the infinity is 0 , and let $\mathcal{K}_{*}\left(\mathbb{R}^{N}\right)=\mathcal{K}_{+}\left(\mathbb{R}^{N}\right)$ be the set of nonnegative continuous functions with compact support. If $*$ is the increasing rearrangement with respect to $v \in$ $\mathbb{R}^{N} \cap \partial B(0,1)$, let

$$
\begin{aligned}
\mathrm{L}_{*}^{p}\left(\mathbb{R}^{N}\right)=\left\{u: \mathbb{R}^{N} \rightarrow[0,1] \mid\right. & \exists h: \mathbb{R} \rightarrow \mathbb{R} \text { such that } h \text { is increasing, } \\
& \left.\left(h-\chi_{\mathbb{R}^{+}}\right) \in \mathrm{L}^{p}(\mathbb{R}), \text { and }(h(v \cdot .)-u) \in \mathrm{L}^{p}\left(\mathbb{R}^{N}\right)\right\},
\end{aligned}
$$

$\mathrm{C}_{*}\left(\mathbb{R}^{N}\right)=\left\{u: \mathbb{R}^{N} \rightarrow[0,1] \mid u\right.$ is continuous,

$$
\left.\lim _{v \cdot x \rightarrow-\infty} u(x)=0, \text { and } \lim _{v \cdot x \rightarrow+\infty} u(x)=1\right\},
$$

and

$$
\begin{aligned}
\mathcal{K}_{*}\left(\mathbb{R}^{N}\right)=\{u \in & \mathrm{C}_{*}\left(\mathbb{R}^{N}\right) \mid \exists h: \mathbb{R} \rightarrow \mathbb{R} \text { such that } h \text { is increasing, } \\
& \left.\left(h-\chi_{\mathbb{R}^{+}}\right) \text {has compact support, and }(h(v \cdot .)-u) \in \mathcal{K}\left(\mathbb{R}^{N}\right)\right\} .
\end{aligned}
$$

The functions of the sets $\mathcal{K}_{*}\left(\mathbb{R}^{N}\right), \mathrm{C}_{*}\left(\mathbb{R}^{N}\right)$, and $\mathrm{L}_{*}^{p}\left(\mathbb{R}^{N}\right)$ are all admissible. If $u \in \mathcal{K}_{*}\left(\mathbb{R}^{N}\right)$, then $u^{*} \in \mathcal{K}_{*}\left(\mathbb{R}^{N}\right)$ and, for any $c \in \mathbb{R}$,

$$
\{u>c\}^{*}=\left\{u^{*}>c\right\} .
$$

The preservation of inclusions (2.1) and measure (2.2) imply that the symmetrization of functions is nonexpansive for any $\mathrm{L}^{p}$ norm, $1 \leq p \leq \infty$. The ideas of Crowe, Zweibel and Rosenbloom [7, and of Alberti [1] can be generalized to embrace the case of the increasing rearrangement.

Proposition 2.14. For any $1 \leq p \leq \infty$, we have

$$
\left\|u^{*}-v^{*}\right\|_{p} \leq\|u-v\|_{p} .
$$

Proof. If $1 \leq p<\infty$, for any admissible functions $u$ and $v$, we have

$$
\begin{aligned}
\int_{\Omega}|u-v|^{p} d x=\int_{\sigma \leq \tau}\left(\mathcal{L}^{N}(\right. & \{v>\tau\} \backslash\{u>\sigma\}) \\
& \left.+\mathcal{L}^{N}(\{u>\tau\} \backslash\{v>\sigma\})\right) p(p-1)|\sigma-\tau|^{p-2} d \sigma d \tau .
\end{aligned}
$$

The properties (2.4) and (2.3) bring the conclusion. If $p=\infty$, the conclusion follows from the preservation of inclusions.

\section{Polarizations}

Definition 3.1. A polarizer is a closed affine halfspace of $\mathbb{R}^{N}$.

Remark 3.2. A set $H$ is a polarizer if and only if there exists $a \in \mathbb{R}^{N},|a|=1$ and $b \in \mathbb{R}^{N}$ such that $H=\left\{x \in \mathbb{R}^{N} \mid a \cdot x \geq b\right\}$. 
Notation 3.3. If $x \in \mathbb{R}^{N}$ and $H \subseteq \mathbb{R}^{N}$ is a polarizer, $x_{H}$ denotes the reflection of $x$ with respect to $\partial H$. Using the description of Remark 3.2, $x_{H}=x-2(a \cdot x-b) a$.

Definition 3.4. The polarization of a function $u: \mathbb{R}^{N} \rightarrow \mathbb{R}$ by a polarizer $H$ is the function $u^{H}: \mathbb{R}^{N} \rightarrow \mathbb{R}$, defined by

$$
u^{H}(x)= \begin{cases}\max \left\{u(x), u\left(x_{H}\right)\right\} & \text { if } x \in H, \\ \min \left\{u(x), u\left(x_{H}\right)\right\} & \text { if } x \notin H .\end{cases}
$$

Remark 3.5. The polarization is also called two-point rearrangement. The polarization by $H$ is the natural extension of the cap symmetrization with respect to $S=H$ when $\operatorname{dim} S=N$ (compare with Definition 2.4).

Notation 3.6. If $T$ is an affine subspace, let

$$
\mathcal{H}_{T}=\left\{H \subset \mathbb{R}^{N} \mid H \text { is a polarizer, and } T \subset H\right\},
$$

if $S$ is a closed half affine subspace, let

$$
\mathcal{H}_{S}=\left\{H \subset \mathbb{R}^{N} \mid H \text { is a polarizer, } S \subset H, \text { and } \partial S \subset \partial H\right\}
$$

and, if $v \in \mathbb{R}^{N} \cap \partial B(0,1)$, let

$\mathcal{H}_{(v, \infty)}=\left\{H \subset \mathbb{R}^{N} \mid H\right.$ is a polarizer, and $a=v$ in the description of Remark 3.2 $\}$.

For any symmetrization $*$, and for any function $u: \mathbb{R}^{N} \rightarrow \mathbb{R}$,

$$
u=u^{*} \quad \Longleftrightarrow \quad \forall H \in \mathcal{H}_{*}, u=u^{H} .
$$

Polarizations satisfy the properties (2.1) and (2.2). Thus they are nonexpansive. For $u \in \mathrm{L}_{*}^{p}\left(\mathbb{R}^{N}\right)$ and $H \in \mathcal{H}_{*}$, the inequality

$$
\left\|u^{H}-u^{*}\right\|_{p}=\left\|u^{H}-\left(u^{*}\right)^{H}\right\|_{p} \leq\left\|u-u^{*}\right\|_{p}
$$

suggests that well-chosen polarization can approximate the symmetrization $*$ for a given function. The proof goes in two steps: first the relative compactness of any sequence of iterated polarizations is established (Lemma 3.7), then a convergence condition ensures the convergence to the symmetrized function (Lemma 3.9).

Lemma 3.7. Let $u \in \mathcal{K}_{*}\left(\mathbb{R}^{N}\right)$ and $\left(H_{m}\right)_{m \geq 0} \subset \mathcal{H}_{*}$ be a sequence of polarizers. Let $u_{m}=u^{H_{1} \cdots H_{m}}$. Then, there is $v \in \mathcal{K}_{*}\left(\mathbb{R}^{N}\right)$ and an increasing sequence $\left(m_{k}\right)_{k \in \mathbb{N}}$ in $\mathbb{N}$ such that, for any $1 \leq p \leq \infty$,

$$
\lim _{k \rightarrow \infty}\left\|v-u_{m_{k}}\right\|_{p}=0 .
$$

Remark 3.8. This lemma is essentially due to Brock and Solynin [5, lemmas 6.1 and 6.2], and the main part of the arguments was given by Baernstein [3]. Smets and Willem proved it for the cap symmetrization [12.

Proof. The compactness of the sequence $\left(u_{m}\right)_{m>1}$ is proven by Ascoli-Arzelá's theorem. The sequence is equibounded: for any polarization $H,\left\|u^{H}\right\|_{\infty}=\|u\|_{\infty}$ and thus, by induction, $\left\|u_{m}\right\|_{\infty}=\|u\|_{\infty}<+\infty$.

Second, the sequence is equicontinuous. Let

$$
\omega_{v}(\delta)=\sup \{v(x)-v(y) \mid d(x, y) \leq \delta\}
$$

be the modulus of continuity of a function $v$. By a tedious analysis of the possible different cases, it can be proved that for any polarization $H, \omega_{u^{H}} \leq \omega_{u}$, and thus, 
by induction, $\omega_{u_{m}} \leq \omega_{u}$. Since $u \in \mathcal{K}_{*}\left(\mathbb{R}^{N}\right)$ is uniformly continuous, the sequence is equicontinuous.

It remains to prove that the supports are uniformly bounded. For the Steiner or the cap symmetrizations, $u \in \mathcal{K}_{*}\left(\mathbb{R}^{N}\right)$ implies that, for some $p$ in $T$ or in $\partial S$, and for some $R>0,\{u>0\} \subseteq B(p, R)$. Thus, because polarizations are monotone, $\left\{u^{H}>0\right\} \subseteq B(p, R)^{H}=B(p, R)$ and, by induction, $\left\{u_{m}>0\right\} \subseteq B(p, R)$.

For the increasing rearrangement with respect to $v$, we have, for some $c \in \mathbb{R}$,

$$
\{u>0\} \subseteq\left\{x \in \mathbb{R}^{N} \mid v \cdot x>c\right\}
$$

and

$$
\left\{u^{H}>0\right\} \subseteq\left\{x \in \mathbb{R}^{N} \mid v \cdot x>c\right\}^{H}=\left\{x \in \mathbb{R}^{N} \mid v \cdot x>c\right\} .
$$

Therefore we have

$$
\left\{u_{m}>0\right\} \subseteq\left\{x \in \mathbb{R}^{N} \mid v \cdot x>c\right\}
$$

and similarly there exists $d \in \mathbb{R}$ such that

$$
\left\{x \in \mathbb{R}^{N} \mid v \cdot x>d\right\} \subseteq\left\{u_{m}<1\right\} .
$$

There exists $R>0$ such that $u_{m}(x) \neq h(x)$ implies $\operatorname{dist}(x, x+v \mathbb{R}) \leq R$. Therefore, there is a bounded set $B \subset \mathbb{R}^{N}$ such that $\operatorname{supp}\left(u_{m}-h\right) \subset B$ for $m \in \mathbb{N}$. We conclude, by Arzela-Ascoli's theorem, that any subsequence has a subsequence converging uniformly to some $v \in \mathcal{K}_{*}\left(\mathbb{R}^{N}\right)$.

The convergence for $1 \leq p<+\infty$ follows from the convergence for $p=+\infty$ and from the fact that all the supports of the functions of the sequence $\left(u_{m}-v\right)$ lie in the same compact set.

A second lemma states that for any nonsymmetrical function, there exist a polarizer $H \in \mathcal{H}_{*}$ that brings it closer to its symmetrization.

Lemma 3.9. Let $u \in \mathcal{K}_{*}\left(\mathbb{R}^{N}\right)$. If $u \neq u^{*}$, then there is a polarizer $H \in \mathcal{H}_{*}$ such that, for any $1 \leq p<+\infty$,

$$
\left\|u^{H}-u^{*}\right\|_{p}<\left\|u-u^{*}\right\|_{p} .
$$

Remark 3.10. This lemma is due to Brock and Solynin [5] for the Steiner symmetrization and to Smets and Willem [12 for the cap symmetrization.

Proof. Since $u \neq u^{*}$, there exists $c>0$ such that the set $\{u>c\} \Delta\left\{u^{*}>c\right\}$ is not empty. Choose a point $y \in\left\{u^{*}>c\right\} \backslash\{u>c\}$. There is a polarizer $H \in \mathcal{H}_{*}$ such that $y_{H} \in\{u>c\} \backslash\left\{u^{*}>c\right\}$. In a sufficiently small neighborhood $N \subset H$ of $y$, we then have

$$
u^{H}(x)=u\left(x_{H}\right)>c \geq u^{*}\left(x_{H}\right) \quad \text { and } \quad u^{*}(x)>c \geq u(x)=u^{H}\left(x_{H}\right),
$$

whence, for $p \geq 1$,

$$
\left|u(x)-u^{*}(x)\right|^{p}+\left|u\left(x_{H}\right)-u^{*}\left(x_{H}\right)\right|^{p}>\left|u^{H}(x)-u^{*}(x)\right|^{p}+\left|u^{H}(x)-u^{*}\left(x_{H}\right)\right|^{p} .
$$

If $x \in N$, the corresponding nonstrict inequality holds. The integral inequality is obtained by integration of the preceding inequality over $N$ and of the nonstrict inequality on $H \backslash N$. 


\section{Approximation by polarizations}

We first establish the convergence of a sequence of polarizations for a single function.

Lemma 4.1. Let $u \in \mathcal{K}_{*}\left(\mathbb{R}^{N}\right), 0<\kappa<1,\left(m_{k}\right)_{k \geq 1} \subset \mathbb{N}$ be an increasing sequence of indices, and a sequence of polarizers $\left(H_{m}\right)_{m \geq 1} \subset \mathcal{H}_{*}$ such that for all $k \in \mathbb{N}$,

$$
\left\|u_{m_{k}}-u^{*}\right\|_{1}-\left\|u_{m_{k}}{ }^{H_{m_{k}}}-u^{*}\right\|_{1} \geq \kappa \sup _{H \in \mathcal{H}_{*}}\left(\left\|u_{m_{k}}-u^{*}\right\|_{1}-\left\|u_{m_{k}}{ }^{H}-u^{*}\right\|_{1}\right) .
$$

Then the sequence $u_{m}=u^{H_{1} \ldots H_{m}}$ converges to $u^{*}$ for any $1 \leq p \leq+\infty$.

Remark 4.2. For any function $u \in \mathcal{K}_{*}\left(\mathbb{R}^{N}\right)$, a sequence of polarizers verifying condition (4.1) can be constructed.

Remark 4.3. We use the same strategy of proof as that of Smets and Willem [12, except that the inequality (4.1) is weaker than imposing $\left(H_{m}\right)$ to be optimal as done in [12].

Proof. By Lemma 3.7, there exist a subsequence $u_{m_{k}^{\prime}}$ of $\left(u_{m_{k}}\right)_{k \geq 1}$ that converges to $v \in \mathcal{K}_{*}$ for any $\mathrm{L}^{p}$ norm. Since the rearrangement $*$ is nonexpansive,

$$
\left\|u^{*}-v^{*}\right\|_{p}=\lim _{k \rightarrow \infty}\left\|u_{m_{k}^{\prime}}{ }^{*}-v^{*}\right\|_{p} \leq \lim _{k \rightarrow \infty}\left\|u_{m_{k}^{\prime}}-v\right\|_{p}=0
$$

and $v^{*}=u^{*}$. For any polarizer $H \in \mathcal{H}_{*}$, we then have, by the nonexpansiveness of polarizations and by equation (4.1),

$$
\begin{aligned}
& \left\|u_{m_{k+1}^{\prime}}-u^{*}\right\|_{1} \leq\left\|u_{m_{k}^{\prime}+1}-u^{*}\right\|_{1} \\
& \leq\left\|u_{m_{k}^{\prime}}-u^{*}\right\|_{1}+\kappa\left(\left\|u_{m_{k}^{\prime}}{ }^{H}-u^{*}\right\|_{1}-\left\|u_{m_{k}^{\prime}}-u^{*}\right\|_{1}\right) \\
& \quad=(1-\kappa)\left\|u_{m_{k}^{\prime}}-u^{*}\right\|_{1}+\kappa\left\|u_{m_{k}^{\prime}}{ }^{H}-u^{*}\right\|_{1} \leq\left\|u_{m_{k}^{\prime}}-u^{*}\right\|_{1} .
\end{aligned}
$$

Passing to the limit, we obtain

$$
\left\|v-u^{*}\right\|_{1} \leq(1-\kappa)\left\|v-u^{*}\right\|_{1}+\kappa\left\|v^{H}-u^{*}\right\|_{1} \leq\left\|v-u^{*}\right\|_{1},
$$

whence, since $u^{*}=v^{*},\left\|v-v^{*}\right\|_{1}=\left\|v^{H}-v^{*}\right\|_{1}$, which is absurd if $v \neq u^{*}$ By Lemma 3.9. Therefore the subsequence $\left(u_{m_{k}^{\prime}}\right)_{k \in \mathbb{N}}$ converges to $u^{*}$ for any $\mathrm{L}^{p}$ norm. The nonexpansiveness of polarizations allows us to conclude that

$$
\lim _{k \rightarrow \infty}\left\|u_{k}-u^{*}\right\|_{p} \leq \lim _{k \rightarrow \infty}\left\|u_{m_{k}^{\prime}}-u^{*}\right\|_{p}=0 .
$$

Theorem 4.4. For any symmetrization $*$, there exist a sequence of polarizers $\left(H_{m}\right)_{m \geq 0} \subset \mathcal{H}_{*}$ such that, for any $1 \leq p<\infty$, if $u \in \mathrm{L}_{*}^{p}\left(\mathbb{R}^{N}\right)$, the sequence

$$
u_{m}=u^{H_{1} \cdots H_{m}}
$$

converges to $u^{*}$ :

$$
\lim _{m \rightarrow \infty}\left\|u_{m}-u^{*}\right\|_{p}=0
$$

If $u \in \mathrm{C}_{*}\left(\mathbb{R}^{N}\right)$, the sequence converges for $p=\infty$.

Proof of Theorem 4.4. If $*$ is a Steiner or spherical cap symmetrization, first note that there is a countable set $N \subset \mathcal{K}_{*}\left(\mathbb{R}^{N}\right)$ dense in $\mathrm{L}_{*}^{p}\left(\mathbb{R}^{N}\right)$ and in $\mathrm{C}_{*}\left(\mathbb{R}^{N}\right)$ (see [13). Choose a sequence $\left(H_{m}\right)$ for which (4.1) holds for all $u \in N$. The sequence of iterated polarizations approaches the symmetrization for any $u \in N$. 
Let $u \in \mathrm{L}_{*}^{p}\left(\mathbb{R}^{N}\right)$ and $\epsilon>0$. By density, there is $v \in N$ such that $\|u-v\|_{p} \leq \epsilon / 3$. By contraction, for $m$ sufficiently large, if $v_{m}=v^{H_{1} \cdots H_{n}}$, $\left\|u_{m}-u^{*}\right\|_{p} \leq\left\|u_{m}-v_{m}\right\|_{p}+\left\|v_{m}-v^{*}\right\|_{p}+\left\|v^{*}-u^{*}\right\|_{p} \leq 2\|u-v\|_{p}+\left\|v_{m}-v^{*}\right\|_{p} \leq \epsilon$.

If $*$ is the increasing rearrangement with respect to $v$, and $h: \mathbb{R} \rightarrow[0,1]$ is a continuous function such that $\operatorname{supp}\left(h-\chi_{\mathbb{R}^{+}}\right)$is compact, then the same reasoning shows the convergence for any $w \in \mathrm{L}_{*}^{p}\left(\mathbb{R}^{N}\right) \cap\left(h(v \cdot)+.\mathrm{L}^{p}\left(\mathbb{R}^{N}\right)\right)$. Let $u \in \mathrm{L}_{*}^{p}\left(\mathbb{R}^{N}\right)$ and

$$
C_{R}=\left\{x \in \mathbb{R}^{N}||(v \cdot x) v-x \mid \leq R\right\} .
$$

Consider the function $u_{R}$ which is equal to $u$ on $C_{R}$ and equal to $h$ outside of it. Then $u_{R} \in \mathrm{L}_{*}^{p}\left(\mathbb{R}^{N}\right) \cap\left(h(v \cdot)+.\mathrm{L}^{p}\left(\mathbb{R}^{N}\right)\right)$, and thus $\int_{C_{R}}\left|u_{m}-u^{*}\right|^{p} d x \rightarrow 0$. Since

$$
\int_{\mathbb{R}^{N} \backslash C_{R}}\left|u_{m}-u^{*}\right|^{p} d x \leq 2 \int_{\mathbb{R}^{N} \backslash C_{R}}|u-h|^{p} d x
$$

$u_{m} \rightarrow u^{*}$ follows.

The proof is similar for $u \in \mathrm{C}_{*}\left(\mathbb{R}^{N}\right)$.

Remark 4.5. Theorem 4.4 implies that the symmetrization of any set can be approximated in measure and in Hausdorff distance [5. Lemma 7.2]. Conversely, if the symmetrization of any set can be approximated in measure by some fixed sequence of polarizations, then Theorem 4.4 follows by the approximation of functions in $\mathrm{L}^{p}\left(\mathbb{R}^{N}\right)$ by simple functions.

\section{Approximation by Symmetrizations}

The method of proof of Theorem 4.4 can be extended to approximation of Steiner or symmetrizations by lower order Steiner or cap symmetrizations.

Definition 5.1. Let $T$ be an affine subspace. A set of affine subspaces $\mathcal{T}$ approximates $T$ if, for any $T^{\prime} \in \mathcal{T}, T \subset T^{\prime}$, and for any affine subspace $T^{\prime \prime} \subset \mathbb{R}^{N}$ of codimension 1 such that $T \subset T^{\prime \prime}$, there exists $T^{\prime} \in \mathcal{T}$ such that $T^{\prime} \subset T^{\prime \prime}$.

Theorem 5.2. Let $T$ be an affine subspace of $\mathbb{R}^{N}$ and let $\mathcal{T}$ be a set of affine subspaces. If $\mathcal{T}$ approximates $T$, there is a sequence $\left(T_{m}\right)_{m \geq 1}$ in $\mathcal{T}$ such that $u^{T_{1} \cdots T_{m}} \rightarrow u^{T}$ for $u \in \mathrm{L}_{+}^{p}\left(\mathbb{R}^{N}\right)$ or $u \in \mathrm{C}_{0}\left(\mathbb{R}^{N}\right)$.

Definition 5.3. Let $T$ be an affine subspace. A set $\mathcal{S}$ of closed half affine subspaces of $\mathbb{R}^{N}$ approximates $T$ if, for any $S^{\prime} \in \mathcal{S}, T \subset S^{\prime}$, and for any affine subspace $T^{\prime \prime} \subset \mathbb{R}^{N}$ of codimension 1 which is parallel to $T$, there exists $S^{\prime} \in \mathcal{S}$ such that $\partial S^{\prime} \subset T^{\prime \prime}$.

Example 5.4. If $\mathcal{T}=\{T\}$, then $\mathcal{T}$ trivially approximates $T$. If $T=\{0\}$, the set of polarizers $\mathcal{H}_{T}$ and the set of closed halflines containing 0 both approximate $T$.

Definition 5.5. Let $S$ be a closed half affine subspace. A set $\mathcal{S}$ of closed half affine subspaces of $\mathbb{R}^{N}$ approximates $S$ if, for any $S^{\prime} \in \mathcal{S}, S \subset S^{\prime}$ and $\partial S \subset S^{\prime}$, and for any affine subspace $T^{\prime \prime} \subset \mathbb{R}^{N}$ of codimension 1 which is parallel to $T$, there exists $S^{\prime} \in \mathcal{S}$ such that $\partial S^{\prime} \subset T^{\prime \prime}$.

Theorem 5.6. Let $T$ be an affine subspace of $\mathbb{R}^{N}$ (resp. let $S$ be a half affine subspace of $\mathbb{R}^{N}$ ) and let $\mathcal{S}$ be a set of closed half affine subspaces of $\mathbb{R}^{N}$. If $\mathcal{S}$ approximates $T$ (resp. $S$ ), then there exists a sequence $\left(S_{m}\right)_{m \geq 1}$ in $\mathcal{S}$ such that $u^{S_{1} \cdots S_{m}} \rightarrow u^{T}\left(\right.$ resp. $\left.u^{S_{1} \cdots S_{m}} \rightarrow u^{S}\right)$ for $u \in \mathrm{L}_{+}^{p}\left(\mathbb{R}^{N}\right)$ or $u \in \mathrm{C}_{0}\left(\mathbb{R}^{N}\right)$. 
Proof. The proofs of Theorems 5.2 and 5.6 are similar. The proof is essentially the same as the proof of Theorem 4.4. The modifications in the lemmas are sketched for a closed half affine subspace $S$ in Theorem [5.6 Suppose $u \in \mathcal{K}_{+}\left(\mathbb{R}^{N}\right)$. It is clear that for any $S^{\prime} \in \mathcal{S}, u^{S S^{\prime}}=u^{S}$ and $\left\|u^{S^{\prime}}-u^{S}\right\|_{p} \leq\left\|u-u^{S}\right\|_{p}$. Therefore the sequence $\left\|u^{S_{1} \ldots S_{n}}-u^{S}\right\|_{p}$ is nonincreasing. Theorem 4.4 implies that the modulus of continuity decreases along the sequence. This allows us to prove an analogue to Lemma 3.7. An analogue of Lemma 3.9 is also needed. Suppose $u \neq u^{S}$. Then by Lemma 3.9 there exists $H \in \mathcal{H}_{S}$ such that $\left\|u^{H}-u^{S}\right\|_{p}<\left\|u-u^{S}\right\|_{p}$. Since $\partial H$ is parallel to $\partial S$ and $\mathcal{S}$ approximates $S$, there exists $S^{\prime}$ such that $S^{\prime} \subset H$ and $\partial S^{\prime} \subset \partial H^{\prime}$. Hence $u^{H S^{\prime}}=u^{S^{\prime}}$ and

$$
\left\|u^{S^{\prime}}-u^{S}\right\|_{p} \leq\left\|u^{H}-u^{S}\right\|_{p}<\left\|u-u^{S}\right\|_{p} .
$$

The remainder of the proof is the same as the proof of Lemma 4.1 and of Theorem 4.4 .

\section{Pólya-Szegö's Inequality}

Definition 6.1. A set $\Omega$ is totally invariant with respect to a symmetrization $*$ if for any $H \in \mathcal{H}_{*}, \Omega$ is invariant under the reflection with respect to $\partial H$.

Definition 6.2. If $*$ is a symmetrization, $\Omega$ is a totally invariant set and $u: \Omega \rightarrow \mathbb{R}$ is a function, then the symmetrization of $u$ is $u^{*}=\left.\tilde{u}^{*}\right|_{\Omega}$, where $\tilde{u}$ is any extension of $u$ to $\mathbb{R}^{N}$.

The definition of the symmetrization of $u: \Omega \rightarrow \mathbb{R}$ does not depend on the extension $\tilde{u}$ because $\Omega$ is totally invariant.

Corollary 6.3. If $\Omega$ is a totally invariant open set, $*$ is a Steiner or cap symmetrization, $u \in \mathrm{W}_{\text {loc }}^{1,1}(\Omega)$ is admissible, $1<p<+\infty$, and $\nabla u \in \mathrm{L}^{p}(\Omega)$, then

$$
\left\|\nabla u^{*}\right\|_{p} \leq\|\nabla u\|_{p} .
$$

Proof. Suppose first that $u \in \mathrm{L}_{*}^{p}(\Omega)$. Let $u_{m}$ be the restrictions to $\Omega$ of the sequence of iterated polarizations of Theorem 4.4 applied to an extension $\tilde{u} \in \mathrm{L}_{*}^{p}\left(\mathbb{R}^{N}\right)$ of $u$ to $\mathbb{R}^{N}$. For any compactly supported smooth function $h \in \mathcal{D}(\Omega)^{N}$,

$$
\begin{aligned}
&-\int_{\Omega} u^{*} \operatorname{div} h d x=-\lim _{m \rightarrow \infty} \int_{\Omega} u_{m} \operatorname{div} h d x=\lim _{m \rightarrow \infty} \int_{\Omega} \nabla u_{m} h d x \\
& \leq \liminf _{m \rightarrow \infty}\left\|\nabla u_{m}\right\|_{p}\|h\|_{p^{\prime}}=\|\nabla u\|_{p}\|h\|_{p^{\prime}},
\end{aligned}
$$

since $\left\|\nabla u^{H}\right\|_{\Omega, p}=\|\nabla u\|_{\Omega, p}\left[\underline{5}\right.$ for any $u \in \mathrm{W}_{\text {loc }}^{1,1}(\Omega)$ such that $\nabla u \in \mathrm{L}^{p}\left(\mathbb{R}^{N}\right)$, and for any polarizer $H$. Thus there exist $v \in \mathrm{L}^{p}(\Omega)^{N}$ that is the weak limit of $\nabla u_{m}$ and the weak gradient of $u^{*}$.

In general, if $*$ is an increasing rearrangement, for $m \geq 3$, let

$$
u_{m}(x)=\frac{m}{m-2} \min (\max (0, u(x)-1 / m), 1-2 / m) .
$$

Since $u$ is admissible, $u_{m} \in \mathrm{L}_{*}^{1}(\Omega)$. From the first part of the proof, $\left\|\nabla u_{m}{ }^{*}\right\|_{p} \leq$ $\left\|\nabla u_{m}\right\|_{p}$. Since $\frac{m-2}{m}\left|\nabla u_{m}\right| \nearrow|\nabla u|$ and $\frac{m-2}{m}\left|\nabla u_{m}{ }^{*}\right| \nearrow\left|\nabla u^{*}\right|$ almost everywhere, the conclusion comes from the monotone convergence theorem. The end of the proof is similar for the Steiner and cap symmetrizations. 


\section{ACKNOWLEDGEMENT}

The author thanks Michel Willem for his encouragement and for his careful rereading. He also thanks the anonymous referee for his constructive comments and suggestions.

\section{REFERENCES}

1. G. Alberti, Some remarks about a notion of rearrangement, Ann. Scuola Norm. Sup. Pisa Cl. Sci. (4) 29 (2000), no. 2, 457-472. MR.1784182 (2001k:46050)

2. M. Badiale, Monotonicity of solutions for elliptic systems on unbounded domains, Boll. Un. Mat. Ital. A (7) 6 (1992), no. 1, 59-69. MR1164736 (93g:35037)

3. A. Baernstein, II, A unified approach to symmetrization, Partial Differential Equations of Elliptic Type (A. Alvino et al., eds.), Sympos. Math., no. 35, Cambridge Univ. Press, 1995, pp. 47-49. MR.1297773 (96e:26019)

4. H. J. Brascamp, E. H. Lieb, and J. M. Luttinger, A general rearrangement inequality for multiple integrals, J. Funct. Anal. 17 (1974), 227-237. MR0346109(49:10835)

5. F. Brock and A. Yu. Solynin, An approach to symmetrization via polarization, Trans. Amer. Math. Soc. 352 (2000), no. 4, 1759-1796. MR1695019 (2001a:26014)

6. G. Carbou, Unicité et minimalité des solutions d'une équation de Ginzburg-Landau, Ann. Inst. H. Poincaré Anal. Non Linéaire 12 (1995), no. 3, 305-318. MR1340266 (96m:35078)

7. J. A. Crowe, J. A. Zweibel, and P. C. Rosenbloom, Rearrangements of functions, J. Funct. Anal. 66 (1986), 432-438. MR0839110 (87j:28003)

8. E. H. Lieb and M. Loss, Analysis, second ed., Grad. Stud. Math., vol. 14, American Mathematical Society, Providence, RI, 2001. MR1817225 (2001i:00001)

9. P. Mani-Levitska, Random Steiner symmetrizations, Studia Sci. Math. Hungar. 21 (1986), no. 3-4, 373-378. MR0919382 (89a:52012)

10. G. Pólya and G. Szegö, Isoperimetric inequalities in mathematical physics, Princeton Univ. Press, 1951. MR0043486 (13:270d)

11. J. Sarvas, Symmetrization of condensers in n-space., Ann. Acad. Sci. Fenn., Ser. A I no. 522 (1972), 44 pp. MR0348108(50:606)

12. D. Smets and M. Willem, Partial symmetry and asymptotic behavior for some elliptic variational problems, Calc. Var. Partial Differential Equations 18 (2003), no. 1, 57-75. MR2001882 (2004m:35092)

13. M. Willem, Analyse fonctionnelle élémentaire, Cassini, Paris, 2003.

Département de Mathématiques, Université Catholique de louvain, Chemin du cyClotron 2, B-1348 Louvain-La-Neuve, Belgium

E-mail address: vanschaftingen@math.ucl.ac.be 